\title{
MedienPädagogik
}

Zeitschrift für Theorie und Praxis der Medienbildung

Themenheft Nr. 38: Aneignung politischer Information in Social Media und Internet. Forschungslage und medienpädagogische Implikationen

Herausgegeben von Kai Hugger, Kai Kaspar und Lars Gräßer

\section{Game Designer als Akteure der politischen Bildung}

André Czauderna und Alexandra Budke

\section{Zusammenfassung}

Viele digitale Spiele enthalten - auch wenn sie primär für Unterhaltungszwecke konzipiert wurden - Anknüpfungspunkte für die politische Bildung. So erlauben sie z. B. ihren Spielerinnen und Spielern, in die Rolle politischer Entscheidungsträger zu schlüpfen und sich mit Themen wie Stadtentwicklung, Migration, Ressourcenkonflikte oder Klimawandel zu beschäftigen, welche $u$. a. für die politische Bildung im Geographieunterricht eine wichtige Rolle spielen. Es ist dementsprechend konsequent, ihre Designerinnen und Designer nicht nur als Akteurinnen und Akteure der Kulturindustrie, sondern auch als solche der politischen Bildung anzusehen. Der vorliegende Beitrag rekonstruiert aus medienpädagogischer und geographiedidaktischer Perspektive mithilfe einer qualitativen Inhaltsanalyse von neun leitfadengestützten Interviews mit Game Designerinnen und Designern von Unterhaltungsspielen, wie diese ihre Rolle als Akteurinnen und Akteure der politischen Bildung wahrnehmen. Insbesondere wird herausgearbeitet, dass ihre Haltung zum Design politischer Entscheidungssituationen weitgehend an den Massstäben des Beutelsbacher Konsenses - $d$. h. dem Überwältigungsverbot, dem Kontroversitätsgebot und der Interessenorientierung - gemessen werden kann. Gleichwohl muss der Einsatz kommerzieller Spiele in der politischen Bildung in jedem Einzelfall kritisch reflektiert und pädagogisch begleitet werden. Da sich ihre Designerinnen und Designer in ihrer Rolle als Akteurinnen und Akteure der Kulturindustrie - die keinen Bildungsauftrag, aber Kunstfreiheit umfasst - in erster Linie der Unterhaltung ihrer Zielgruppe verpflichtet fühlen müssen, können ihre Produkte nicht genauso streng nach didaktischen Kriterien bewertet werden wie speziell für die politische Bildung erstellte Materialien. Eine kritische Reflexion der Spiele in der politischen Bildung sollte sich u. a. auch dem von den meisten Designerinnen und Designern selbst gesehenen «Demokratiedefizit» der Spiele, das sich z. B. in der Vernachlässigung der intersubjektiven Aushandlung von Entscheidungen darstellt, widmen.

Game designers as agents of political education

\begin{abstract}
Many digital games designed for entertainment are relevant for political education because they allow their players to slip into roles of political decision-makers and deal with topics such as urban development, migration, resource conflicts or climate change. Thus, game designers can be perceived not only as agents of the culture industry, but
\end{abstract}


also as agents of political education. The present article reconstructs how designers of entertainment games shape this role, from the perspectives of media education and geography education, based on a qualitative content analysis of nine semi-structured interviews. In particular, it is shown that game designers' positions towards the design of political decision-making are largely consistent with the "Beutelsbach Consensus», which constitutes a set of basic principles for political education in Germany. Nevertheless, since entertainment game designers are first and foremost obliged to entertain their players, their products cannot be judged according to educational criteria just as strictly as materials created specifically for political education. Therefore, the use of entertainment games in political education must be accompanied by an in-depth classroom discussion. A critical reflection of digital games in political education should discuss, among other things, their neglect of democratic negotiation processes.

\section{Einführung}

Das Potential digitaler Spieler zur Initiierung von Lern- und Bildungsprozessen wurde in zahlreichen Studien (z. B. Gee 2007; Steinkuehler und Squire 2014), darunter auch solchen aus der deutschsprachigen Medienpädagogik (z. B. Fromme, Jörissen, und Unger 2008) herausgearbeitet. Im medienpädagogischen Diskurs wird mittlerweile nicht mehr nur der schulische Einsatz von digitalen Lernspielen - den sogenannten «Educational Games» oder «Serious Games», also jenen Spielen, die gezielt für pädagogische Zwecke entwickelt wurden -, sondern im Rahmen lebensweltorientierter Ansätze auch die Verwendung von Unterhaltungsspielen diskutiert (z. B. Riemer und Möring 2020; Preisinger und Aumayr 2020). Es wird angenommen, dass Unterhaltungsspiele im Vergleich mit Lernspielen in bestimmten pädagogischen Kontexten in höherem Masse zur Motivation der Lernenden beitragen können und sich darüber hinaus durch anspruchsvollere Lernmechanismen auszeichnen, weshalb ihre Nutzung in der formalen und non-formalen Bildung unter bestimmten Voraussetzungen - je nach Zielsetzung der Lehrenden und bei Reflexion ihrer Schwachstellen z. B. hinsichtlich ihrer fachlichen Korrektheit - gegenüber Lernspielen vorzuziehen sein kann (z. B. Gee 2007; Riemer und Möring 2020).

Auch für die politische Bildung, die in Deutschland eine fächerübergreifende Aufgabe ist, liegen neben Lernspielen (Cuhadar und Kampf 2014; Motyka 2018) etliche geeignete Unterhaltungsspiele vor (Czauderna 2019; 2020; Preisinger und Aumayr 2020). Insbesondere in vielen zur Unterhaltung entwickelten Strategie- und Managementspielen lassen sich typische Themen der politischen Bildung wiederfinden. So befassen sich beispielsweise Spielerinnen und Spieler in Stadtsimulationen, wie Cities: Skylines, mit Stadtplanung und Lokalpolitik, während sie in den Spielen der Democracy- und Tropico-Reihen die Regierung eines Staates übernehmen. Die in diesen Spielen dargestellten Probleme eint, dass sie im Kern auf «polytelischen 
Situationen» basieren, wie sie von Dörner (2002) sowie Betsch, Funke, und Plessner (2011, 142 f.) beschrieben wurden, d. h. also auf Zielkonflikten, wie etwa dem zwischen Wirtschaftswachstum auf der einen Seite und Umweltschutz auf der anderen Seite (Czauderna und Budke 2020). Indem sich die Spielerinnen und Spieler selbst in der Rolle von politischen Entscheidungsträgern mit der Lösung dieser Probleme zu beschäftigen haben, wird ihnen begreiflich gemacht, warum ebendies so schwierig ist und der intensiven gesellschaftlichen Aushandlung bedarf. Insgesamt ermöglichen die Spiele ihren Spielerinnen und Spielern damit, die Entwicklung von Meinungen und Positionen sowie das Ausprobieren verschiedener Handlungsmöglichkeiten, was ein zentrales Lernziel jeder politischen Bildung ist, die ihre Adressaten zu mündigen Bürgerinnen und Bürgern erziehen möchte. Es ist folglich davon auszugehen, dass für Unterhaltungszwecke entwickelte Strategie- und Managementspiele als methodisches Instrument auch zur politischen Bildung im Fach Geographie, insbesondere zum Erwerb von Urteils- und Entscheidungsfähigkeiten beitragen können, die für politisch mündige Bürgerinnen und Bürger unabdingbar sind (Budke 2016, 13).

In der vorliegenden Studie setzen wir uns aus medienpädagogischer und geographiedidaktischer Perspektive mit den Intentionen der Designerinnen und Designer dieser digitalen Spiele auseinander, die wir in ihrer Rolle als Gestalterinnen und Gestalter von politischen Entscheidungssituationen auch als Autorinnen und Autoren didaktischer Materialien für die politische Bildung im Schulfach Geographie wahrnehmen. Insgesamt soll mit der Studie untersucht werden, inwiefern Game Designerinnen und Designer, die politische Entscheidungssituationen in digitalen Unterhaltungsspielen gestalten, unter den gegebenen Bedingungen kommerzieller Produktionszusammenhänge - bewusst oder unbewusst - als Akteurinnen und Akteure der politischen Bildung denken und handeln.

Mit der Berücksichtigung digitaler Spiele als Vermittler politischer Informationen tragen wir dem Umstand Rechnung, dass sich die Aneignung politischer Informationen verändert hat. So beeinflussen neben expliziten Informationsangeboten in Social Media und Internet auch digitale Spiele (off- und online) die politische Meinungsbildung und Sozialisation. Aus medienpädagogischer Sicht gilt es dies nicht nur zu problematisieren (wenn z. B. in kommerziellen Shooter-Spielen kriegerische Konflikte unangemessen dargestellt oder sogar verherrlicht werden), sondern auch als Chance zu begreifen, wenn beispielsweise in Politiksimulationen Spielerinnen und Spieler mit politischen Positionen experimentieren können und in diesem Zusammenhang interessante informelle Lernprozesse zu beobachten sind (z. B. Czauderna 2019), was prinzipiell auch für die Gestaltung von Lern- und Bildungsprozessen (z. B. Czauderna 2020) nutzbar gemacht werden kann.

Die Verortung der Studie in Medienpädagogik und Geographiedidaktik korrespondiert mit den unterschiedlichen disziplinären Hintergründen der Autorin und des Autors des vorliegenden Beitrages. Die Medienpädagogik lenkte unseren Blick 
auf die Bildungspotentiale digitaler Spiele, die einen besonderen Stellenwert in der Lebenswelt von Kindern und Jugendlichen einnehmen. Die Geographiedidaktik bestimmte den Fokus der Studie auf die Themen Klimawandel, Migration, nachhaltige Stadtentwicklung und Ressourcenkonflikte. Zugleich legte sie den fachdidaktischen Rahmen und das konkrete Anwendungsfeld fest. Gleichwohl gehen wir davon aus, dass unsere Überlegungen grundsätzlich auch auf die Fachdidaktiken der anderen gesellschaftswissenschaftlichen Fächer übertragbar sind.

Im Rahmen unserer Studie führten wir neun leitfadengestützte Interviews mit Game Designerinnen und Designern von erfolgreichen kommerziellen digitalen Spielen, die inhaltlich anschlussfähig an zentrale Themen der politischen Bildung im Fach Geographie sind. Daraufhin analysierten wir ihre Äusserungen zum Design politischer Entscheidungssituationen in ihren Spielen mit den Mitteln der qualitativen Inhaltsanalyse (Mayring 2010) und auf der Basis des Beutelsbacher Konsenses (Wehling 1977), an dem sich politische Bildung im Geographieunterricht orientiert. ${ }^{1}$

\section{Theoretische Perspektive}

Der Überlegung, dass Strategie- und Managementspiele auf Zielkonflikten mit Relevanz für Themen des Geographieunterrichts wie Stadtplanung, Migration, Klimawandel und Ressourcenkonflikte basieren, folgend, untersuchten wir in einer vorherigen Studie, inwiefern diese Form von Unterhaltungsspielen ihren Spielerinnen und Spielern die Erprobung und Reflexion von Entscheidungen in komplexen und dynamischen Situationen, die für die politische Bildung im Geographieunterricht bedeutsamen sind, erlauben (Czauderna und Budke 2020). In diesem Zusammenhang entwickelten wir ein theoretisches Modell der Gestaltung von Entscheidungsprozessen in Strategie- und Managementspielen, welches wir mithilfe einer qualitativen Auswertung von 17 Spielen veranschaulichten.

Im Rahmen der vorliegenden Studie schauen wir nicht mehr nur auf die beschriebenen Entscheidungssituationen selbst, sondern erweitern den Blick auf die Perspektive bzw. Rolle ihrer Urheberinnen und Urheber unter besonderen Berücksichtigung ihrer impliziten Agenda der politischen Bildung.

Die folgende Konzeptualisierung betrifft Spiele, die aktuelle politische Themen aufgreifen, welche in demokratischen Gesellschaften intensiv und kontrovers diskutiert werden und die im Geographieunterricht behandelt werden. Das im Verlauf dieses Kapitels entwickelte präskriptive Modell dient uns als Ausgangspunkt für die im

1 Wir gehen davon aus, dass ein Einsatz digitaler Spiele in der politischen Bildung nur dann sinnvoll ist, wenn diese den Grundsätzen des Beutelsbacher Konsenses entsprechen oder geeignet sind, diese Grundsätze anhand der Spiele im Unterricht zu thematisieren. Dies gilt umso mehr als die Designerinnen und Designer von Unterhaltungsspielen dem Beutelsbacher Konsens erst einmal überhaupt nicht verpflichtet sind und Arbeiten der Game Studies darauf verweisen, dass digitale Spiele als programmierte Artefakten bzw. Algorithmen grundsätzlich Ideologien beinhalten können und zur Überwältigung und politischen Manipulation eingesetzt werden können (Bogost 2007). 
Rahmen dieser Studie durchgeführte qualitative Inhaltsanalyse von Interviews mit den Designerinnen und Designern solcher Spiele. Das Modell stellt ein idealtypisches Entscheidungsdesign dar, das sowohl lerntheoretischen als auch fachdidaktischen Ansprüchen gerecht werden kann.

Die fachspezifische Aufgabe des Geographieunterrichts im Kontext der politischen Bildung liegt «in der Offenlegung der Beziehungen zwischen Gesellschaft und Raum und in der Entwicklung von Mündigkeit zu ihrer Analyse und Bewertung sowie zur verantwortungsbewussten Gestaltung von Räumen» (Budke 2016, 16). Im Unterricht werden diese Ziele u. a. durch die Prinzipien des Perspektivenwechsels sowie der Konflikt- und Problemorientierung umgesetzt. Diese Prinzipien beruhen u. a. auf dem Beutelsbacher Konsens (Wehling 1977). Im Kern besteht dieser aus den folgenden drei Grundsätzen: (1) die Adressaten politischer Bildung sollen nicht indoktriniert werden (dem Überwältigungsverbot), (2) die Adressaten politischer Bildung sollen das ganze Spektrum kontroverser Positionen kennenlernen (dem Kontroversitätsgebot), (3) die Adressanten politischer Bildung sollen lernen, politische Situationen auf Grundlage ihrer eigenen Interessenlage zu analysieren und auch politische Interessen wahrzunehmen bzw. die politische Lage gemäss der eigenen Interessen zu beeinflussen. D. h. es soll politische Handlungskompetenz gefördert werden (Interessenorientierung).

Wenngleich der Beutelsbacher Konsens, d. h. z. B. auch das Kontroversitätsgebot, heute - mehr als 40 Jahre nach seiner Entstehung - u. a. im Zusammenhang mit den Wahlerfolgen rechtspopulistischer oder sogar rechtsextremistischer Parteien durchaus kontrovers diskutiert wird (z. B. Widmaier und Zorn 2016), stellt er nach wie vor eine wichtige Referenz verschiedener Fachdidaktiken dar, darunter auch die der Geographiedidaktik (Budke 2016). Daher knüpfen auch wir an seine Grundsätze an, wenn wir im Folgenden unser oben genanntes Modell (Czauderna und Budke 2020) um die Ebene der politischen Bildung erweitern (siehe Abb. 1). 


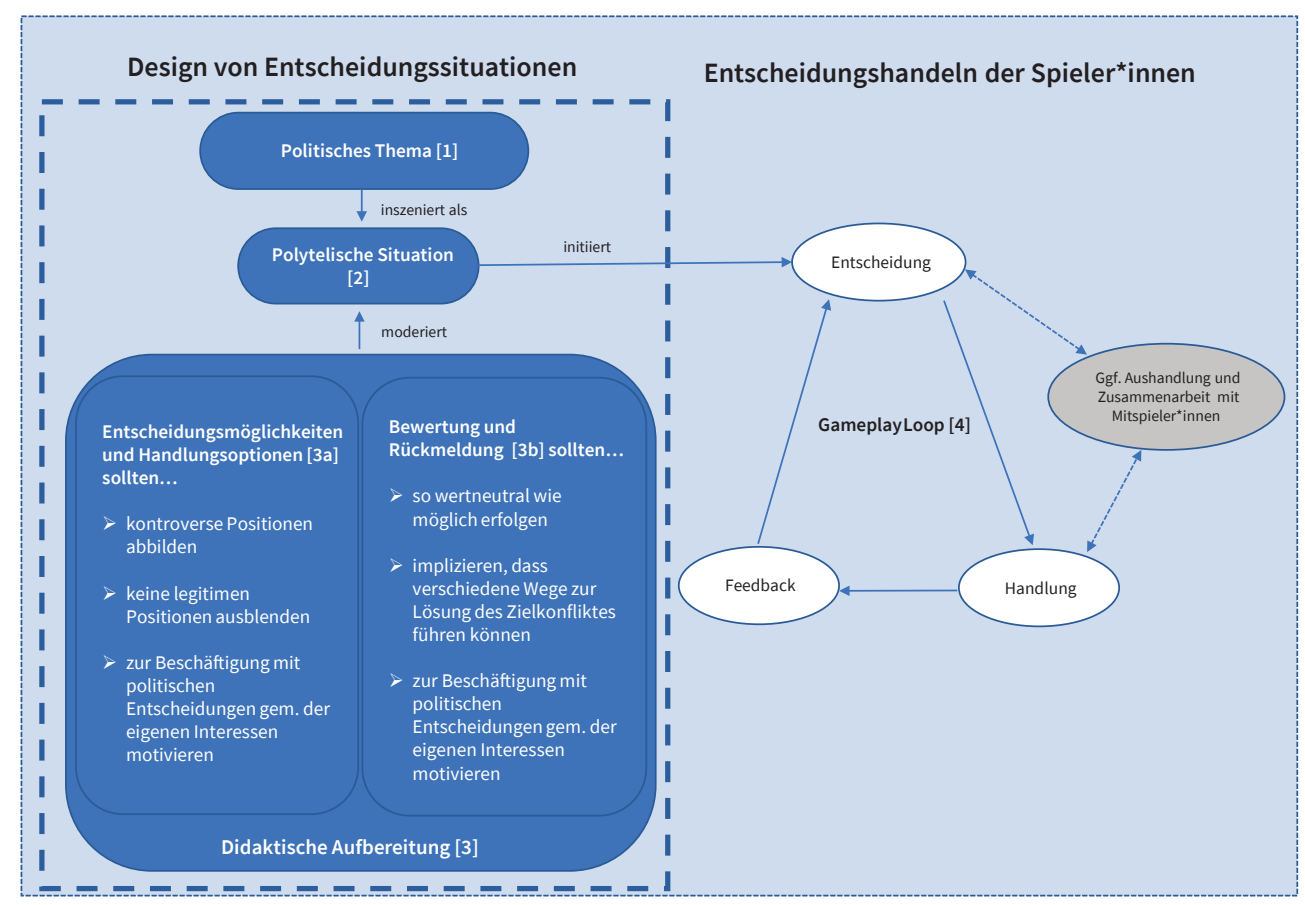

Abb. 1.: Modell des Designs von Entscheidungssituationen in digitalen Spielen für die politische Bildung nach den Massstäben des Beutelsbacher Konsenses (Eigenes Modell: André Czauderna und Alexandra Budke).

Politische Themen, die in der Gesellschaft ausserhalb des Spiels diskutiert werden [1], sollten als polytelische Situationen [2], d. h. als Zielkonflikte, inszeniert werden, die das jeweilige Thema im Sinne des Beutelsbacher Konsenses so vollständig, komplex, vielschichtig und kontrovers wie in einer Simulation möglich abbilden. Die erforderliche Strittigkeit, die politischen Themen innewohnt, wird also zunächst durch das Vorhandensein der polytelischen Situationen erzeugt, die z. B. auch diverse Positionen von zentralen Akteurinnen und Akteuren abbilden sollten.

Zur Lösung dieser Konflikte schlüpfen die Spielerinnen und Spieler in kommerziellen Spielen dieser Art in die Rolle eines - in der Regel unrealistisch mächtigen - Entscheidungsträgers. Diese Rolle verlangt von ihnen die aktive Beschäftigung mit den politischen Entscheidungssituationen. In der Sprache des Game Design initiiert die polytelische Situation damit einen «Gameplay Loop» [4] (Salen und Zimmerman 2003, 316; Guardiola 2016; Czauderna und Guardiola 2019), der das Handeln der Spielerinnen und Spieler in Interaktion mit dem Spiel als zirkuläres Verhältnis zwischen Entscheidung, Handlung und Feedback modelliert. Damit erhalten sie die Gelegenheit, die Konsequenzen und Komplexität politischer Entscheidungen kennenzulernen und im Idealfall ihre eigenen Entscheidungen kritisch zu bewerten und im weiteren Verlauf im Sinne der Spiellogik anzupassen. 
Wenngleich die Spielerinnen und Spieler ihre Entscheidungen in den meisten der in unserer vorherigen Studie (Czauderna und Budke 2020) untersuchten Spiele alleine treffen (d. h. sie verhandeln ihre Entscheidungen weder mit simulierten Agenten - sog. NPCs, non-player characters - in Form von politischen Akteurinnen und Akteuren noch mit ihren Mitspielerinnen und Mitspielern) und die Spiele damit ein «Demokratiedefizit» aufweisen, muss das hier vorgestellte präskriptive Modell die denkbare Möglichkeit zum gemeinsamen Entscheiden und Handeln im Rahmen eines Multiplayer-Spieles enthalten. Die Modellierung des «Gameplay Loop» ist daher um die Dimension der Kommunikation und Kollaboration mit anderen Spielerinnen und Spielern zu ergänzen - auch wenn diese in den Analysen der vorliegenden Studie eine untergeordnete Rolle spielt, weil der Grossteil der u. a. aufgrund des thematischen Bezuges zum Fach Geographie ausgewählten Spiele auf einem EinzelspielerModus basiert.

Für die weitere Bewertung des Designs der Entscheidungssituationen nach den Massstäben des Beutelsbacher Konsenses ist insbesondere die didaktische Aufbereitung der polytelischen Situationen [3] entscheidend. Denn wenn die Game Designerinnen und Designer im Zuge der didaktischen Vermittlung den Rahmen zur Lösung der Zielkonflikte aufspannen, können sie ihre Spielerinnen und Spieler zum einen motivieren, zum anderen aber auch beeinflussen. An dieser Stelle ist im Wesentlichen zwischen zwei Formen der didaktischen Aufbereitung zu unterscheiden. So werden zum einen Entscheidungsmöglichkeiten bzw. Handlungsoptionen vorgegeben [3a]. Zum anderen erfolgt eine Bewertung und Rückmeldung zu den jeweils gewählten Optionen, d. h. Entscheidungen und Handlungen [3b].

Damit verbunden ist grundsätzlich eine Einschränkung der Freiheiten der Spielerinnen und Spieler und die Gefahr der (gewollten oder ungewollten) Persuasion (Bogost 2007). So werden den Spielerinnen und Spielern eben nicht alle real denkbaren Handlungsmöglichkeiten angeboten. Und da es auch um das Gewinnen oder Verlieren geht, werden bestimmte Entscheidungen stärker honoriert als andere Entscheidungen. Game Designerinnen und Designer beeinflussen ihre Spielerinnen und Spieler zudem durch deutliches Feedback, so dass vollständige Autonomie nicht erreicht werden kann und der Selbstbestimmung und Selbstgestaltung Grenzen gesetzt sind. All dies muss besonders im Hinblick auf Kinder und Jugendliche problematisiert werden und erscheint in Anbetracht der grossen Bedeutung von Identifikation, Involvement und Immersion bei der Rezeption digitaler Spiele umso wichtiger.

Um nun den Massstäben des Beutelsbacher Konsenses gerecht zu werden, sollten die Entscheidungsmöglichkeiten und Handlungsoptionen [3a] kontroverse Positionen abbilden; keine legitimen Positionen ausblenden; und zur Beschäftigung mit politischen Entscheidungen gemäss der eigenen Interessen motivieren. 
Die Bewertung der Entscheidungen und Handlungen der Spielerinnen und Spieler sowie die damit verbundene Rückmeldung des Spieles [3b] sollten dann im Sinne des Beutelsbacher Konsenses so wertneutral wie möglich erfolgen; implizieren, dass verschiedene Wege zur Lösung des Zielkonfliktes führen können; und die Beschäftigung mit politischen Entscheidungen gemäss der eigenen Interessen weiter forcieren.

Zusammenfassend kann bezogen auf die drei Kriterien des Beutelsbacher Konsenses festgehalten werden: (1.) Zur Lösung der Zielkonflikte in polytelischen Situationen muss im Rahmen der didaktischen Vermittlung ein breites Spektrum kontroverser Entscheidungsmöglichkeiten bzw. Handlungsoptionen aufgespannt werden; der Entscheidungs- und Handlungsspielraum muss möglichst gross sein (Kontroversitätsgebot). (2.) Die didaktische Aufbereitung darf nicht zu einer Überwältigung führen. Daher sollten keine politisch diskutierten und legitimen Entscheidungs- und Handlungsoptionen ausgespart bleiben und alle im Spiel möglichen Entscheidungen und Handlungen sollten gleichermassen Erfolg im Spiel erlauben (Überwältigungsverbot). (3.) Der ausgelöste "Gameplay Loop» sollte den Spielerinnen und Spielern ermöglichen, spielerisch politisches Handeln zu praktizieren, d. h. vor allem politische Entscheidungen zu vollziehen und dabei Selbstwirksamkeit und Mitbestimmung zu erleben. Wenn auch (1.) und (2.) gewährleistet ist, bekommen die Spielerinnen und Spieler die Möglichkeit, ihr Handeln an den eigenen Interessen auszurichten. So werden sie zur Teilhabe an realen politischen Prozessen und Einbringung eigener Interessen ermutigt (Interessenorientierung). Der «Gameplay Loop» mit dem ihm innewohnenden Kreislauf von Entscheidung und Handlung einerseits und Feedback andererseits wirkt dabei idealerweise motivierend und lernförderlich (Czauderna und Budke 2020).

An dieser Stelle sei zur Verdeutlichung erläutert, was unseres Erachtens Spiele ausmacht, die den oben erläuterten didaktischen Leitlinien nicht entsprechen: sie weisen einen geringen Handlungs- und Entscheidungsspielraum auf; übervorteilen bestimmte Entscheidungen, $d$. h. Erfolg im Spiel ist nur mit bestimmten Entscheidungen möglich (was den Spielerinnen und Spielern durch Feedback rückgemeldet wird); und die Spielerrolle ist sehr begrenzt, was mit einer geringen Selbstwirksamkeitserfahrung einhergehen kann. Nichtsdestotrotz kann in der politischen Bildung auch eine Analyse dieser Spiele, darunter auch «persuasive games», die von politischen Akteurinnen und Akteuren zur Überzeugung eingesetzt werden (Bogost 2007), Sinn machen - vorausgesetzt sie erfolgt in kritischer Absicht. 


\section{Methoden im Forschungsprozess}

Im Rahmen der vorliegenden Studie führten wir leitfadengestützte qualitative Interviews mit Repräsentantinnen und Repräsentanten von sieben Spielen (Age of Empires II, Anno 1800, Anno 2070, Bury me, my Love, Democracy 3, Rise of Industry, Eco). Die Vertreter von drei weiteren Spielen (Cities in Motion 2, Cities: Skylines, Tropico 6) reichten ihre Antworten auf unsere Interviewfragen in schriftlicher Form ein. Die Antworten zu den Spielen Cities in Motion 2 und Cities: Skylines, die vom selben Studio entwickelt worden sind, wurden von unserem Kontakt in einem Dokument zusammengefasst. Alle in den neun Interviews behandelten Spiele beschäftigen sich mit einem oder mehreren der für den Geographieunterricht wichtigen, politisch relevanten Themen Stadtplanung, Migration, Klimawandel und Ressourcenkonflikte.

Wir gehen davon aus, dass unsere Interviewpartnerinnen und -partner in den Interviews nicht nur ihre eigene Position vertreten, sondern auch für die Gruppe der an der Spielentwicklung beteiligten Kolleginnen und Kollegen sprechen und damit über die eigene Meinung hinausweisend eine kollektive Haltung des jeweiligen Teams wiedergeben. ${ }^{2}$

Die Auswertung der Interviewtranskripte und schriftlichen Antworten erfolgte in Form der qualitativen Inhaltsanalyse (Mayring 2010) mit Hilfe der Software MAXQDA auf Grundlage eines Kategoriensystems, das auf einer Kombination deduktiver und induktiver Kategorienentwicklung basiert. Ein Grossteil der Kategorien konnte aus dem oben dargestellten Modell abgeleitet werden.

\section{Inhaltsanalysen}

Im Folgenden fassen wir die Ergebnisse unserer qualitativ-inhaltsanalytischen Auswertung der neun Interviews zusammen.

\subsection{Motive für die Wahl realer politischer Themen als Spielgegenstand}

Die von uns interviewten Designerinnen und Designer sind alle an Spielen beteiligt, die reale politische Themen behandeln wie Klimawandel, Migration, nachhaltige Stadtentwicklung oder Ressourcenkonflikte. Es stellt sich jetzt die Frage, aus welchen Gründen diese realen Themen gewählt wurden und ob das jeweilige Spiel als Beitrag zur politischen Aushandlung verstanden wird.

Zu diesen Fragestellungen unterscheiden sich die Darlegungen der Befragten. Ein Teil der Interviewten ist sich über den politischen Gehalt ihrer Spiele nur teilweise im

2 Der sprachlichen Einfachheit halber verwenden wir im Rahmen des Beitrages überwiegend den Begriff Game Designerinnen bzw. Game Designer, meinen damit aber auch die beiden interviewten Producer (von Cities in Motion 2 und Cities: Skylines sowie von Tropico 6) und den Community Manager (von Eco). In unseren Interviews hatten wir es mehrheitlich mit männlichen Interviewpartnern zu tun. Nur im Fall von Anno 1800 sprachen wir - in unserem einzigen Gruppeninterview - mit zwei weiblichen Personen. 
Klaren. Einige Designerinnen und Designer waren von politischen Diskussionen der Spieler zu Aspekten ihrer Spiele überrascht, wie hier in Bezug auf Cities: Skylines:

«We were quite surprised to notice a backlash from assumed global warming denialists for taking a political stance with Green Cities. The purpose was not to make a statement on environmental issues, but just bring new content to the game with a current theme.» (Cities: Skylines und Cities in Motion 2)

Politische Themen werden in den Spielen teilweise vor allem gewählt, um das Interesse der Spieler zu wecken:

«Wir haben das Thema 2070 mit dem Klimawandel dann tatsächlich gewählt, weil wir das Gefühl hatten okay, das Thema berührt und eine gewisse Realitätsnähe ist auch durchaus für den Kunden interessant, um auch einen stärkeren Bezug dazu zu haben.» (Anno 2070).

Dabei werden in der Regel diejenigen politischen Themen gewählt, die aktuell besonders stark gesellschaftlich diskutiert werden, die populär sind und bei denen die Entwicklerinnen und Entwickler daher besonders grosses Interesse erwarten.

Ein anderer Teil der Interviewten berichtet dagegen, dass sehr bewusst reale und aktuelle politische Themen für die Spiele gewählt wurden. Eine Motivation ist dabei, dass die Öffentlichkeit informiert werden soll und man durch die Spiele einen Beitrag zur politischen Meinungsbildung leisten möchte.

Nach Angaben des Designers von Bury me, my Love wurde das Thema «Migration» gezielt als Spielthema verwendet, um über die Schicksale von Migrantinnen und Migranten aufzuklären und ihre Perspektive zu vermitteln. Das Spiel wird als politisches Statement im Diskurs zum Thema aufgefasst, der diese Sichtweise zu wenig berücksichtigen würde:

«I think that in the context of how we as European[s] discuss international forced migrations, we very often tend to completely forget about the human part of the question [...] So my relationship as a designer to this question is, if I can help people to have a more diverse representation of this question of migrations, [then] it's something that I can bring to the table, and I think it's useful.» (Bury me, my Love)

Neben der Popularität bestimmter in den Spielen angesprochener Themen ist für die Wahl politischer Inhalte teilweise auch die politische Meinung und die Interessen der Game Designerinnen und Designer relevant. So wird das Thema «Klimawandel» 
nach Angaben des Designers von Democracy ${ }^{3}$ darum gewählt, da es für den Designer persönlich besonders wichtig ist:

«And to be honest, again, when that [Klimawandel] was put in the game, that wasn't as big an issue as it is now, that was mostly put in the game because I care about it enormously.» (Democracy)

Letztendlich gibt es auch Designerinnen und Designer, die versuchen, politische Themen möglichst wenig in ihren Spielen zu behandeln und die politischen Diskussionen um und durch ihre Spiele vermeiden wollen. Aus diesem Grund wurde z. B. das Spiel Rise of Industry in die 1930er-Jahre verlegt und es wurden Umweltthemen ausgespart:

«I really just want people to enjoy a management game without looking at messages that I put [there] for political purposes, I'm just making a game. So that is why it was pushed from the modern era to the 1930s, so there would be this complete disconnection like no talking about the current climate, this is a game, stop it.» (Rise of Industry).

Von diesem Interviewten wird die Aufgabe von Spielen primär in der Unterhaltung der Spielerinnen und Spieler gesehen, bei der politische Inhalte möglicherweise ablenken oder abzulehnen sind, da sie unterschwellig politisch beeinflussen könnten.

Teilweise werden auch politische Themen in den Spielen ausgespart, da man sich nicht in der Lage sieht, deren politische Komplexität und Kontroversität im Spiel abzubilden, wie das bei dem Thema «Sklaverei» in Anno 1800 der Fall ist.

\subsection{Wahrgenommenes Verhältnis von Unterhaltung und politischer Bildung}

Wenngleich die befragten Designerinnen und Designer ihre Spiele für Unterhaltungszwecke entwickelt haben, sprechen sie ihnen - auf Nachfrage - ein Potential für die fächerübergreifende politische Bildung zu. Einige Game Designerinnen und Designer gehen sogar davon aus, dass es gerade der Zugang über die Unterhaltung ist, der Vorteile für das Lernen mit sich bringen kann. So hofft der Democracy-Designer, dass es sich bei seinem Spiel um ein «subtle [...] stealth teaching tool» handelt, das seines Erachtens der speziell für schulische Kontexte entwickelten «edutainment software» überlegen sei, weil Lernen besser funktioniere, wenn es von etwas ausgehe, wie z. B. einem Spiel, das dem Lernenden schon an sich Freude bereitet anstatt von etwas, wie eben «Edutainment», das primär pädagogische Ziele verfolgt:

3 Das Interview mit dem Designer der Democracy-Reihe bezog sich zunächst auf Democracy 3 aus dem Jahre 2013. Allerdings befand sich der Designer zum Zeitpunkt des Interviews im Jahre 2020 mitten in der Entwicklung der neuen Version, Democracy 4. Grundsätzlich gehen wir davon aus, dass die meisten seiner Aussagen für beide Spiele gültig sind. 
"l've always thought the kind of edutainment software is kind of rubbish, it's got something it wants to teach you and it tries to pretend it's fun, and I think it works much better the other way around, where you've got something that is inherently fun, and you go actually can we also cram a lot of interesting information [about history or politics] that people will learn as they play it, I think that works so much better.» (Democracy)

Trotz des grundsätzlichen Potentials, das die Designerinnen und Designer ihren Spielen für die politische Bildung zuschreiben, betonen sie die Grenzen ihrer Spiele, die primär Unterhaltungszwecken dienen, wenn es um das Ziel der politischen Bildung geht, deutlich. Unter anderem verweisen sie auf Probleme, die aus der Reduktion der Komplexität der abgebildeten Systeme und der Ausgestaltung der Spielerrolle inklusive des damit verbundenen spielimmanenten «Demokratiedefizites» resultieren.

Der Designer von Anno 2070 sagt in diesem Zusammenhang: «Also der Konflikt ist tatsächlich, dass das im Spiel alles sehr simplifiziert und einfach erscheint, während die Realität um ein Vielfaches komplexer ist.» Damit bezieht er sich nicht nur auf eine Vereinfachung von komplexen Zusammenhängen, sondern auch auf fiktionale Lösungen: «Wir hatten im Spiel einen Ozon-Maker, der die Luft gereinigt hat, dann baut man ein paar davon und der Klimawandel ist gelöst». Die damit verbundenen Gefahren für die politische Bildung sind ihm bewusst: «dass vielleicht dann die Wertvorstellung dann da ist, dass Lösungen einfach sind, obwohl sie es nicht sind.»

Die Game Designerinnen und Designer sprechen in den Interviews auch an, dass die Rollen der Spielerinnen und Spieler in ihren Spielen nicht mit real existierenden Rollen politischer Akteurinnen und Akteure in liberalen Demokratien vergleichbar sind. Sie sind sich darüber im Klaren, dass damit ein «Demokratiedefizit» verbunden ist, weil die Spielerinnen und Spieler in der Regel - mit Ausnahme des Spiels ECo - ihre Entscheidungen nicht diskutieren und aushandeln müssen. Die Designerinnen und Designer wissen also um den Mangel an Repräsentation demokratischer Entscheidungsprozesse. Der Interviewpartner zu den Spielen Cities: Skylines und Cities in Motion 2 beschreibt die Rolle seiner Spielerinnen und Spieler als Mischung zwischen Bürgermeister, Stadtrat und Gott. In der Realität jedoch seien für etwas, das die Spielerinnen und Spieler in einem Augenblick bestimmen, etliche Entscheidungsebenen nötig:

"The player is the mayor, the city council, and essentially the god looking over all aspects of the city. In the real world there are several layers of decision-making needed for what the player is able to decide in an instant in the games.» (Cities: Skylines und Cities in Motion 2) 
Der Designer der Democracy-Spiele beschreibt dieses Defizit in der Repräsentation politischer Prozesse ebenfalls. Obwohl er zur Kenntnis genommen hat, dass sich manche Spielerinnen und Spieler ein Mehr an politischer Aushandlung wünschen (weshalb das neue Spiel - Democracy 4 - nun auch eine simple Form des «coalitionbuilding» enthält), verteidigt er letztendlich den überwiegenden Verzicht auf solche Prozesse mit den Logiken von Spielen bzw. dem Unterhaltungserleben der Spielerinnen und Spieler.

\subsection{Beschreibung und Bewertung polytelischer Situationen}

Die befragten Game Designerinnen und Designer schreiben den polytelischen Situationen in ihren Spielen, d. h. den von ihnen inszenierten Zielkonflikten, eine besondere Rolle für das Game Design und damit auch eine besondere Bedeutung für den Spielspass zu. Sie nehmen an, dass ihre Spielerinnen und Spieler grosse Freude daran haben, diese Konflikte zu lösen, indem sie auf Grundlage von strategischen Abwägungen Entscheidungen treffen, d. h. auch verschiedene Handlungsoptionen erwägen und die Folgen möglicher Entscheidungen berücksichtigen. Der Age of Empires-Designer beschreibt die Essenz seines Spieles als einen Prozess des Abwägens und Ausbalancierens: «making trade-offs and making rich decisions and having multiple things to juggle at once and keeping those in balance and keeping those always in mind». Nach den Befragten sind es die Zielkonflikte, welche die Herausforderung und damit auch den Spielspass des Genres ausmachen. Ein Beispiel für einen solchen Konflikt geben die Tropico 6-Produzenten: «Es ist z. B. sehr herausfordernd ein Paradies für Touristen mit stark die Umwelt verschmutzender Ölindustrie zu kombinieren.»

Der Designer der Democracy-Reihe ist davon überzeugt, dass die Lösung politischer Zielkonflikte keineswegs langweilig, sondern besonders spannend ist, weil hier alle - auch budgetäre - Entscheidungen einen grossen Einfluss haben und auch mit Todesfällen als unerwünschten Nebenfolgen einhergehen können. Er illustriert dies an den Beispielen von Intensivstationen und verkehrsberuhigenden Massnahmen, die nicht finanziert werden können, wenn das Geld an anderer Stelle investiert wird: "You know you are making decisions that have a big impact. And I always think that it's interesting to think about the fact that almost every decision, no matter how boring, in government, affectively kills some people, because anytime you're spending money on this thing, you're not spending on intensive care wards, or better traffic calming measures. Indirectly, indirectly, indirectly, someone will die because of what you did. You may save other people, but it is a real serious decision. And to me that makes it interesting.» (Democracy) 


\subsection{Bedeutung von Kontroversität aus Sicht der Spieledesigner}

Um die politische Bildung z. B. im Geographieunterricht umzusetzen, ist das Kontroversitätsgebot des Beutelsbacher Konsenses von besonderer Bedeutung. Es stellt sich jetzt die Frage, inwiefern die Game Designerinnen und Designer kommerzieller Spiele tatsächlich die gesellschaftliche Kontroversität der von innen in den Spielen behandelten Themen berücksichtigen wollen. Tatsächlich wird die inhaltliche Kontroversität in den geführten Interviews nicht direkt thematisiert, allerdings betonen alle Befragten, dass sie ihren Spielern möglichst viele unterschiedliche Handlungsoptionen anbieten wollen. Dies drückt sich auch in dem folgenden Zitat aus: «We really wanted you to feel like you had a lot of choices.» (Age of Empires)

Das Design von vielen verschiedenen Arten, in denen das jeweilige Spiel gespielt und teilweise auch gewonnen werden kann, wird von den Interviewten vorwiegend damit begründet, dass man unterschiedliche Spielertypen, mit ihren jeweiligen Interessen und Vorlieben ansprechen möchte, indem man ihnen die Freiheit bietet, sich selbst darzustellen und auszudrücken, wie auch in diesem Zitat deutlich wird: «So, also the freedom of representing and expressing yourself is also a very important aspect to get players to motivate themselves internally.» (Anno 1800)

Dabei werden im Spiel Democracy 3 auch die möglichen politischen Ausrichtungen der Spielerinnen und Spieler berücksichtigt, wenn ihnen bewusst ermöglicht wird, das Spiel sowohl mit linksgerichteten als auch mit rechtsgerichteten Politiken erfolgreich zu gestalten. Dies führe, den Annahmen des Game Designers zufolge, dazu, dass er sich von allen Seiten mit dem Vorwurf der politischen Voreingenommenheit konfrontiert sehe:

«Well, I mean there's so many ways, there's so many ways to do well in the game. I mean, we've checked that you can play aiming to be very left-wing, and you can win election after election after election, and that you can do the same if you're right-wing, and you can do the same [as] liberal or conservative. And we get told by everyone that there is bias one way or the other, because, you know, it's impossible to get it right. But I don't think there's any.» (Democracy 3 )

Eine in den Interviews angesprochene Strategie, unterschiedliche Handlungsmöglichkeiten zu designen, besteht in der Bereitstellung von gegensätzlichen Charakteren, mit denen die Spielerinnen und Spieler das Spiel durchleben können. Diese können für unterschiedliche gesellschaftliche Gruppen stehen, wie im Fall von Anno 2070:

"Wir haben uns dann dazu entschlossen, dass es zwei Fraktionen geben soll, mit den Ecos und den Tycoons - die Techs gibt's auch noch, aber die sind da eher die neutralere Instanz - auf jeden Fall die Ecos und die Tycoons, zwei total unterschiedliche Ideologien, wie man mit dem Thema Klimawandel umgeht, 
und mit diesem neuen Setting in der Zukunft, und die einen versuchen, den Klimawandel aufzuhalten und sehr umweltbewusst zu leben, nämlich die Ecos, und die Tycoons sind in dem Bild tatsächlich dann eher die Konservativen, die nach dem aktuellen Vorbild einfach weiter wirtschaften und hoch produktiv sind und die die Umwelt nicht weiter beachten.» (Anno 2070)

Die Bandbreite an Handlungsmöglichkeiten, die designt werden, kann dazu führen, dass viele Spielerinnen und Spieler Interesse an dem Spiel entwickeln, sich angesprochen fühlen und dass damit die Verkaufszahlen der Spiele steigen. Dem einzelnen Spieler wird dann durch die regelmässig zu treffenden Entscheidungen zwischen konträren Handlungsmöglichkeiten in polytelischen Situationen möglicherweise vor Augen geführt, dass es sich um eine Thematik handelt, zu der konträre Meinungen und Werte vorliegen. Zudem führt der Wiederholcharakter aller Spiele dazu, dass Spielerinnen und Spieler unterschiedliche Charaktere und ihre Handlungsmöglichkeiten nacheinander ausprobieren können, so dass im Laufe der Zeit ein komplettes Bild der kontroversen Thematik entstehen kann.

Nur in einem von uns geführten Interview werden Diskussionen unter Spielerinnen und Spielern als Simulation von kontroversen Positionen in gesellschaftlichen Aushandlungsprozessen angesprochen. Im Multiplayer-Modus von Eco müssen sich die Spielerinnen und Spieler gemeinsam auf für alle geltende Gesetze einigen, was durch Abstimmung entschieden wird. Dabei wird die Kontroversität der Thematiken besonders deutlich und demokratische Prozesse werden simuliert.

\subsection{Wahrgenommene Beeinflussung der Spielerinnen und Spieler}

Vor dem Hintergrund des Beutelsbacher Konsenses - d. h. insbesondere des Überwältigunsverbotes - ist aus Sicht der politischen Bildung auch zu fragen, inwiefern die Designerinnen und Designer davon ausgehen, ihre Spielerinnen und Spieler zu beeinflussen.

Die qualitativ-inhaltsanalytische Analyse der Interviews zeigt, dass die Game Designerinnen und Designer grundsätzlich den Anspruch erheben, ihren Spielerinnen und Spielern einen grossen Entscheidungsspielraum zu überlassen und sie eben nicht in die eine oder andere Richtung zu drängen. Die Designerinnen und Designer betonen deutlich, dass die Entscheidungen der Spielerinnen und Spieler keineswegs durch eine weltanschauliche Bewertung ihrerseits eingeschränkt werden sollen. So z. B. der interviewte Designer von Anno 2070:

«Jetzt speziell leiten im Sinne von einer Entscheidung, ob jetzt etwas gut oder böse ist und welche Konsequenzen das nach sich zieht, solange es im Regelwerk erlaubt ist, leiten wir glaub ich nicht unbedingt an, weil wir tatsächlich gerade erst mit 2070, mit den zwei verschiedene Fraktionen war's uns wichtig, 
dass die sich unterschiedlich spielen und dass es nicht wirklich ein Gut und Böse gibt, sondern dass der Spieler das nach seinen eigenen Präferenzen spielen kann und da leiten wir ihn nicht in die ein oder andere Richtung, was ist besser, was ist schlechter.» (Anno 2070)

Auch der Designer der Democracy-Serie glaubt, dass er seine Spielerinnen und Spieler nicht beeinflusst oder sogar manipuliert. Im Gegenteil: weltanschauliche bzw. politische Neutralität, die Vermeidung eine Position einzunehmen («without taking a position»), stellt für ihn ein wichtiges Ziel seines Game Designs dar, über das er sich detaillierte Gedanken gemacht hat, wie schon das Zitat im Unterkapitel zur Bedeutung von Kontroversität belegt. Dementsprechend werden in Democracy sogenannte «Achievements», d. h. virtuelle Preise bzw. Urkunden, für ganz unterschiedliche - teils konträre - Errungenschaften, die auf ganz unterschiedliche - teils konträre - Entscheidungen der Spieler zurückzuführen sind, vergeben. So gebe es z. B. solche «Achievements» sowohl für die Unterdrückung als auch die Förderung von religiösem Glauben. Dahinter steht auch die Annahme des Democracy-Designers, dass es seine Zielgruppe nicht mag, wenn sie «überwältigt» wird: «And I don't think players like playing a game where they've been told they're a bad person.» Auch die Tropico -Macher verweisen auf die besondere Bedeutung der Entscheidungsfreiheit, die sie u. a. mit einem Verzicht auf Beeinflussung ermöglichen. Sie räumen allerdings ein, dass ein auf Algorithmen basierendes Spiel, bei dem es auch um das Gewinnen oder Verlieren geht, nicht umhin kommt, den Spieler in irgendeiner Form zu leiten: "Da er zum Gewinnen des Spiels Kriterien erfüllen muss, wird der Spieler dadurch auch durch das Spiel geleitet.» Ausserdem reflektieren die Tropico-Entwickler die Möglichkeit einer vonseiten der Game Designerinnen und Designer, als Schöpfer der Algorithmen, unbewusst erfolgenden Manipulation ihrer Spielerinnen und Spieler: «Unbewusste Manipulation erfolgt dadurch, dass die Definition der Schwere und Art von Konsequenzen durch Menschen erfolgt. Diese lassen (unter)bewusst ihr Weltbild in das Spiel einfließen. Plakativ gesagt: Sind z. B. Soldaten Helden die das Land schützen oder vom Staat legitimierte Mörder? Welcher Grad an Umweltverschmutzung hat keine nennenswerten gesundheitlichen Konsequenzen für die Bevölkerung?» (Tropico 6).

Letztlich gesteht auch der Designer von Democracy ein, dass er gelegentlich seine eigene weltanschauliche Position einfliessen lässt und damit die Entscheidungen der Spielerinnen und Spieler beeinflusst. So z. B. beim Thema «Klimaschutz»: «because I'm an environmentalist, massively». 


\subsection{Motivation zur aktiven Auseinandersetzung mit politischen Entscheidungssituationen}

Es verwundert nicht, dass Designerinnen und Designer digitaler Spiele sehr genau verbalisieren können, wie sie ihre Spielerinnen und Spieler motivieren, da dies zu ihren Kernaufgaben gehört. Bei den interviewten Designerinnen und Designern umfasst das auch eine Reflexion darüber, wie sie die Spielerinnen und Spieler dazu bringen, sich im Spiel - im Sinne der im Beutelsbacher Konsens geforderten Interessenorientierung - aktiv mit politischen Entscheidungssituationen auseinanderzusetzen.

Eine besondere Rolle schreiben die Befragten hier der Handlungsorientierung digitaler Spiele zu: die Spielerinnen und Spieler übernehmen selbst die Rolle der politisch Handelnden, anstelle diese - wie in anderen Medien - nur zu beobachten. Der Designer der Democracy-Serie charakterisiert die Handlungsaufforderung seiner Spiele und den damit verbundenen intrinsischen Motivationsmechanismus mit Bezug auf das Flow-Konzept folgendermassen:

"Loads of things are going wrong, how can you stay on top of everything at once, because if you can you get a sense of kind of flow, a sense of yeah this is impossible, but I've got this, I'm in charge.» (Democracy)

Die Entscheidungen in ihren Spielen werden von den Designerinnen und Designern vor allem auch deshalb als interessant angesehen, weil sie den Spielerinnen und Spielern strategische Abwägungsprozesse abverlangen: «it's trade-offs» (Democracy). Diese kognitiven Aspekte des Spielspasses haben wir bereits im Unterkapitel zu den polytelischen Situationen erläutert. Darüber hinaus spielen aber auch affektive Aspekte eine Rolle. So sind interessante Entscheidungen dem Designer von Anno 2070 zufolge vor allem solche, die für die Spielerinnen und Spieler von Relevanz sind, weil sie emotional berühren und deutliche Konsequenzen zeitigen:

"Also tatsächlich sind immer die Entscheidungen [interessant], glaube ich, wo man emotional aufgewühlt ist, wo man tatsächlich Konsequenzen hat, wo man das Gefühl hat, okay, das ist jetzt etwas, was etwas essenziell verändert.» (Anno 2070)

Die Rolle des emotionalen Involvements betont auch der Designer von Bury me, my Love. Im Vergleich zu den Strategie- und Managementspielen, die in unserem Sample überwiegen, ist hier die Form der emotionalen Betroffenheit noch mal von anderer Qualität, weil der Spieler oder die Spielerin nicht die abstrakte Rolle eines politisch Verantwortlichen, sondern die unmittelbare Perspektive eines Betroffenen - d. h. konkret: die des Ehemannes einer Flüchtenden - einnimmt. In den Strategie- und Managementspielen, in denen die Spielerinnen und Spieler politische Verantwortung übernehmen, resultiert das emotionale Involvement - einigen der befragten Game Designerinnen und Designern zufolge - nicht nur aus der Sorge um 
die durch Spielfiguren und Spielwelt repräsentierten Menschen und Räume, sondern auch aus der eigenen Position zu kontroversen und strittigen Themen, zu denen es sich zu positionieren gilt. Der Democracy-Designer beispielsweise geht davon aus, dass es gerade auch «hot button issues» - also kontroverse strittige Themen wie Abtreibung und Todesstrafe, die stark emotionale Reaktionen hervorrufen - schaffen, die Spielerinnen und Spieler zu involvieren («people get very angry»). Damit tragen sie dazu bei, dass die Spielerinnen und Spieler sich nicht nur mit strittigen Themen beschäftigen, sondern zu ihnen in Form ihrer Entscheidungen unmittelbar Stellung beziehen müssen.

An verschiedenen Stellen in den Interviews verweisen die Interviewpartner zudem auf die besondere Bedeutung der Freiräume ihrer Spiele, die es den Spielerinnen und Spielern ermöglichten, ihren eigenen Präferenzen und Interessen in kontroverse Richtungen zu folgen (wie bereits im Unterkapitel zur Bedeutung von Kontroversität erläutert). Mit dem beschriebenen Spielraum verbunden ist in der Logik digitaler Spiele dann immer auch das Ausprobieren, das Experimentieren, das Herumspielen - hier: mit politischen und ökonomischen Ideen. So sieht sich der Democracy-Designer gerne als Initiator solcher politischer Rollenspiele: «This is economics and this is politics and this is what you can do and you can play around.» (Democracy)

\section{Schlussfolgerungen}

In der vorliegenden Studie haben wir uns aus medienpädagogischer und geographiedidaktischer Perspektive mit der Frage beschäftigt, inwiefern die Designerinnen und Designer von digitalen Unterhaltungsspielen als Akteurinnen und Akteure der politischen Bildung anzusehen sind. Dabei interessierte uns besonders, ob und inwieweit ihre Haltung zum Design politischer Entscheidungssituationen an den Massstäben des Beutelsbacher Konsenses (Wehling 1977) gemessen werden kann.

Insgesamt lässt sich feststellen, dass den Befragten der politische Gehalt ihrer Spiele sehr unterschiedlich bewusst ist. Teilweise werden die politischen Themen nur gewählt, um an «Modethemen» anzuknüpfen und auf diese Weise die Motivation der Spielerinnen und Spieler zu erhöhen. Wenn sich die Designerinnen und Designer des politischen Gehalts ihrer Spiele bewusst sind, fällt dessen Bewertung verschieden aus. Teilweise verstehen sie ihre Spiele als Mittel, um über politische Themen zu informieren und als Beitrag zur gesellschaftlichen Auseinandersetzung. Ein anderer Teil der Befragten versucht den politischen Gehalt der Spiele zu minimieren, indem z. B. die Spiele in Zeiten oder Räume verlegt werden, die nicht aktuell sind und indem kontroverse Unterthematiken ausgespart werden.

Auf Nachfrage können die Designerinnen und Designer das Potential ihrer Spiele für die politische Bildung nicht nur nachvollziehen, sondern auch mit Beispielen belegen. Gleichwohl problematisieren einige Designerinnen und Designer in diesem 
Zusammenhang die für Unterhaltungszwecke notwendige Reduktion von Komplexität (z. B. Gaber 2007, 116), die einem Einsatz in Bildungskontexten entgegenstehen könnte, z. B. weil sie in Form der allmächtigen Spielerrolle mit einem spielimmanenten «Demokratiedefizit» einhergeht (Czauderna 2020).

Die befragten Designerinnen und Designer erheben in der Regel den Anspruch, ihren Spielerinnen und Spielern einen weiten Handlungsspielraum zu bieten und sie möglichst wenig (weltanschaulich) zu beeinflussen - auch wenn einige eingestehen, dass eine gewisse absichtliche oder unabsichtliche Lenkung nicht zu vermeiden ist (z. B. Bogost 2007). Damit ist davon auszugehen, dass sich die Designerinnen und Designer unbewusst darum bemühen, den im Beutelsbacher Konsens formulierten Kontroversitätsgebot und Überwältigungsverbot gerecht zu werden. Dies ist natürlich nicht auf explizit pädagogische Überlegungen zurückzuführen, sondern hängt stark mit Logiken des Game Design zusammen. Hier spielt auch die Berücksichtigung der (vermeintlichen) Bedürfnisse der Spielerinnen und Spieler eine besondere Rolle. Es ist z. B. auffällig, dass die interviewten Game Designerinnen und Designer Kontroversität vorwiegend als Charakteristikum ihrer Zielgruppe begreifen und unterschiedliche Handlungsmöglichkeiten und Spielerrollen bereitstellen möchten, um ein möglichst grosses Publikum zu erreichen. Dabei spielt das Erleben von Kontroversität in kommunikativen Aushandlungssituationen wie im Spiel Eco in den anderen Spielen keine Rolle, was vermutlich damit zu begründen ist, dass unterschiedliche gesellschaftliche Gruppen und ihre Meinungen nur in Ansätzen in den analysierten Spielen simuliert wurden (Lux und Budke 2020) und dass auf die Integration von Multiplayer-Modi, die das gemeinsame Entscheiden mit den Mitspielerinnen und Mitspielern erfordern, verzichtet wurde.

Die im Beutelsbacher Konsens geforderte Interessenorientierung betreffend, kann zusammengefasst werden, dass die Game Designerinnen und Designer eine relativ genaue Vorstellung darüber haben, wie sie ihre Spielerinnen und Spieler dazu motivieren, sich mit politisch relevanten Entscheidungssituationen auseinanderzusetzen - auch wenn sie dies in vielen Fällen nicht direkt mit politischer Bildung in Verbindung bringen. Die Designerinnen und Designer verweisen hier - im Einklang mit der wissenschaftlichen Literatur zum Thema Motivationsmuster digitaler Spiele (z. B. Klimmt 2010) - insbesondere auf die Möglichkeit für die Spielerinnen und Spieler, eine aktiv handelnde Rolle bei der Lösung von politisch relevanten Konflikten einzunehmen, indem sie fortlaufend interessante und wirkmächtige Entscheidungen treffen (Meier 2012). Wenn die Spielerinnen und Spieler mit zahlreichen Herausforderungen gleichzeitig, und insbesondere mit Zielkonflikten in polytelischen Situationen (Dörner 2002; Betsch, Funke, und Plessner 2011, 142 f.), konfrontiert werden, erhalten sie die Gelegenheit, ein Gefühl von Verantwortung und Einfluss zu erfahren sowie den Zustand des «Flow» (Csikszentmihalyi 2009) zu erleben. Die Entscheidungssituationen bewerten die Designerinnen und Designer dann als interessant, wenn sie 
den Spielerinnen und Spielern «Trade-Offs» abverlangen (weil sie auf Zielkonflikten basieren), sie emotional berühren und ihnen Spielräume lassen, den Problemen mit kontroversen Lösungen zu begegnen sowie mit verschiedenen Philosophien und Strategien zu experimentieren (Czauderna 2019). Somit verfolgen die Designerinnen und Designer durchaus eine Interessenorientierung im Sinne des Beutelsbacher Konsenses, die ihren Spielerinnen und Spielern unter bestimmten Bedingungen - z. B. in Verbindung mit einer kritischen Reflexion im Unterricht - dabei helfen kann, auch ausserhalb der virtuellen Welt, und zwar in ihrer eigenen Lebenswelt politisch aktiv zu werden.

Da die Game Designerinnen und Designer - wie gezeigt werden konnte - als Akteurinnen und Akteure der politischen Bildung anzusehen sind, ist ein Einsatz ihrer Spiele in der politischen Bildung im Fach Geographie (genau wie in anderen Schulfächern wie Geschichte, Sozialwissenschaften oder Politik) nur folgerichtig. Allerdings darf dabei nicht vergessen werden, dass die Designerinnen und Designer - als Akteurinnen und Akteure der Kulturindustrie - der künstlerischen Freiheit unterliegen und keinen Bildungsauftrag haben. In ihrer primären Rolle als Game Designerin oder Game Designer fühlen sie sich insbesondere der Unterhaltung (nicht der Bildung) ihrer Zielgruppe verpflichtet. Aus didaktischer Sicht ergeben sich daraus einerseits Vorteile, z. B. im Bereich der Motivation der Adressaten. Andererseits können die Spiele nicht für sich selbst stehen, sondern bedürfen der sorgfältigen Kontextualisierung und kritischen Reflexion, vor allem auch zur bewussten Einhaltung des Beutelsbacher Konsenses. Denn erstens können an die untersuchten Unterhaltungsspiele - auch wenn die Designerinnen und Designer unbewusst die Kriterien des Beutelsbacher Konsenses berücksichtigen - nicht dieselben strengen Massstäbe wie an speziell für die politische Bildung konzipierte Materialien angelegt werden; eine Analyse der Spiele selbst unter Anwendung der strengen Kriterien des Beutelsbacher Konsenses würde zahlreiche problematische Aspekte zutage treten lassen. Und zweitens werden natürlich auch Spiele entwickelt, die ganz bewusst eine politische Message vermitteln wollen, wie z. B. der Democratic Socialism Simulator, in dem die Spielerinnen und Spieler in die Rolle des ersten sozialistischen bzw. sozialdemokratischen Präsidenten der USA schlüpfen und mit rein republikanischen Massnahmen im USamerikanischen Sinne keine Erfolge erzielen werden.

Im Rahmen der erforderlichen kritischen Reflexion im Unterricht sollten auch die Produktionsbedingungen von Unterhaltungsspielen sowie das damit verbundene Spannungsverhältnis zwischen kommerziellen Intentionen und Zwängen einerseits und politischen «Nebeneffekten» oder sogar politischem Anspruch andererseits explizit diskutiert werden. Zudem sollte in Anbetracht der Krise liberaler Demokratien (Stanley 2017; Mounk 2018; Reckwitz 2019, 239 ff.) das herausgearbeitete «Demokratiedefizit» der Spiele, welches sich z. B. in der Vernachlässigung der intersubjektiven Aushandlung von Entscheidungen darstellt, thematisiert werden. Nicht zuletzt bleibt 
zu hoffen, dass in Zukunft die Game Designerinnen und Designer selbst - als Akteurinnen und Akteure der politischen Bildung - die Prozesse politischer Aushandlung von Entscheidungen in ihren Spielen (auch im Multiplayer-Modus) direkt und angemessen simulieren.

\section{Förderhinweis}

Das diesem Artikel zugrunde liegende Forschungsprojekt wurde mit Mitteln des deutschen Bundesministeriums für Bildung und Forschung unter dem Förderkennzeichen 01JD1810A gefördert.

\section{Literatur}

Betsch, Tilmann, Joachim Funke, und Henning Plessner. 2011. Denken - Urteilen, Entscheiden, Problemlösen. Springer-Lehrbuch. Berlin, Heidelberg: Springer. https://doi. org/10.1007/978-3-642-12474-7.

Bogost, Ian. 2007. Persuasive games. Cambridge, MA: MIT Press.

Budke, Alexandra. 2016. «Potentiale der politischen Bildung im Geographieunterricht». In Politische Bildung im Geographieunterricht, herausgegeben von Alexandra Budke, und Miriam Kuckuck, 11-23. Stuttgart: Franz Steiner Verlag.

Csikszentmihalyi, Mihaly. 2009. Flow: The Psychology of Optimal Experience. Nachdr. Harper Perennial Modern Classics. New York: Harper [and] Row.

Cuhadar, Esra, und Ronit Kampf. 2014. «Learning about Conflict and Negotiations through Computer Simulations: The Case of PeaceMaker». International Studies Perspectives 15 (4): 509-24. https://doi.org/10.1111/insp.12076.

Czauderna, André. 2019. «Tinkering with Political Utopias and Dystopias in DEMOCRACY 3. An Educational Perspective». In Playing Utopia, herausgegeben von Benjamin Beil, Gundolf S. Freyermuth, und Hanns Christian Schmidt, 69-98. Bielefeld: transcript Verlag. https://doi. org/10.14361/9783839450505-003.

Czauderna, André. 2020. «Unvollständigkeit als didaktische Chance: Überlegungen zum Einsatz von DEMOCRACY 3 im Politikunterricht». In Videospiele als didaktische Herausforderung, herausgegeben von Nathanael Riemer, und Sebastian Möring, 266-291. Potsdam: Universitätsverlag Potsdam. https://doi.org/10.25932/PUBLISHUP-43074.

Czauderna, André, und Alexandra Budke. 2020. «How Digital Strategy and Management Games Can Facilitate the Practice of Dynamic Decision-Making». Education Sciences 10 (4): 99. https://doi.org/10.3390/educsci10040099.

Czauderna, André, und Emmanuel Guardiola. 2019. «The Gameplay Loop Methodology as a Tool for Educational Game Design». Electronic Journal of e-Learning 17 (3): 207-21. https:// issuu.com/academic-conferences.org/docs/ejel-volume17-issue3-article682. 
Dörner, Dietrich. 2002. Die Logik des Mißlingens: strategisches Denken in komplexen Situationen. 15. Aufl. rororo rororo-Sachbuch rororo science 19314. Reinbek bei Hamburg: Rowohlt.

Fromme, Johannes, Benjamin Jörissen, und Alexander Unger. 2008. «Bildungspotenziale digitaler Spiele und Spielkulturen». MedienPädagogik: Zeitschrift für Theorie und Praxis der Medienbildung 15: 1-23. https://doi.org/10.21240/mpaed/15_16/2008.12.22.X.

Gaber, John. 2007. «Simulating planning: SimCity as a pedagogical tool». Journal of Planning Education and Research 27 (2): 113-21. https://doi.org/10.1177\%2F0739456X07305791.

Gee, James Paul. 2007. What video games have to teach us about learning and literacy. Rev. and Updated ed. New York: Palgrave Macmillan.

Guardiola, Emmanuel. 2016. «The Gameplay Loop: A Player Activity Model for Game Design and Analysis». In Proceedings of the 13th International Conference on Advances in Computer Entertainment Technology - ACE2016, 1-7. Osaka, Japan: ACM Press. https://doi. org/10.1145/3001773.3001791.

Klimmt, Christoph. 2010. Computerspielen als Handlung: Dimensionen und Determinanten des Erlebens interaktiver Unterhaltungsangebote. Unveränd. Nachdr. Unterhaltungsforschung 2. Köln: von Halem.

Lux, Joelle-Denise, und Alexandra Budke. 2020. «Playing with Complex Systems? The Potential to Gain Geographical System Competence through Digital Gaming». Education Sciences 10 (5): 130. https://doi.org/10.3390/educsci10050130.

Mayring, Philipp. 2010. "Qualitative Inhaltsanalyse». In Handbuch qualitative Forschung in der Psychologie, 601-13. Springer. https://doi.org/10.1007/978-3-658-26887-9_52.

Meier, Sid. 2012. «Interesting Decisions (GDC Talk)». Game Developers Conference. https:// www.gdcvault.com/play/1015756/Interesting.

Motyka, Marc. 2018. Digitales, spielbasiertes Lernen im Politikunterricht. Wiesbaden: Springer VS.

Mounk, Yascha. 2018. Der Zerfall der Demokratie: wie der Populismus den Rechtsstaat bedroht. München: Droemer.

Preisinger, Alexander, und Florian Aumayr, Hrsg. 2020. Digitale Spiele im Geschichtsunterricht \& in der Politischen Bildung. Wien: Edition polis.

Reckwitz, Andreas. 2019. Das Ende der Illusionen: Politik, Ökonomie und Kultur in der Spätmoderne. Edition Suhrkamp 2735. Berlin: Suhrkamp.

Riemer, Nathanael, und Sebastian Möring. 2020. Videospiele als didaktische Herausforderung. Potsdam: Universitätsverlag Potsdam.

Salen, Katie, und Eric Zimmerman. 2003. Rules of play: game design fundamentals. Cambridge, Mass: MIT Press.

Stanley, Jason. 2017. How Propaganda Works. First paperback printing. Princeton, New Jersey Oxford: Princeton University Press. 
Steinkuehler, Constance, und Kurt Squire. 2014. «Videogames and Learning». In The Cambridge Handbook of the Learning Sciences, herausgegeben von R. Keith Sawyer, 2. Aufl., 377-94. Cambridge: Cambridge University Press. https://doi.org/10.1017/CB09781139519526.023.

Wehling, Hans-Georg. 1977. «Konsens à la Beutelsbach. Nachlese zu einem Expertengespräch». In Das Konsensproblem in der politischen Bildung, herausgegeben von Siegfried Schiele, und Herbert Schneider, 173-184. Stuttgart: Ernst Klett.

Widmaier, Benedikt, und Peter Zorn. 2016. «Brauchen wir den Beutelsbacher Konsens». Eine Debatte der politischen Bildung. Bonn: Bundeszentrale für politische Bildung. https://m. bpb.de/system/files/dokument_pdf/1793_Beutelsbacher_Konsens_ba.pdf. 